\title{
Age Difference in Morphology and Immunohistology in the Thymus and Spleen in Crl:CD (SD) Rats
}

\author{
Rinya Kodama ${ }^{1}$, Takanobu Okazaki1, Tamio Satoํ, Shuichiro Iwashige ${ }^{1}$, Yohei Tanigawa ${ }^{1}$,

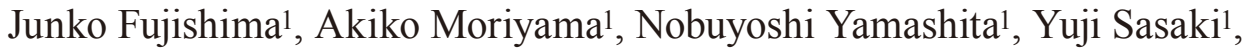 \\ Tsuyoshi Yoshikawa ${ }^{1}$, Yasuhiro Kamimura ${ }^{1}$ and Hiroshi Maeda ${ }^{1}$
}

${ }^{1}$ Drug Safety Research Laboratories, Shin Nippon Biomedical Laboratories, Ltd., 2438 Miyanoura, Kagoshima 891-1394, Japan

\begin{abstract}
We investigated chronological changes in immunohistochemical phenotyping in the thymus and spleen in Crl:CD rats up to the age of about one year. In the thymus, T cells increased markedly from 3 to 4 weeks of age. Proliferating cells also increased markedly at these points. B cells tended towards an increase with age. In the spleen, white pulp increased until 9 weeks of age and remained fairly stable thereafter. In the periarteriolar lymphoid sheath and marginal zone, T cells gradually increased until 9 weeks of age and became almost flat thereafter. In the lymph follicle, T cells increased with age. B cells tended towards an increase with age in all areas of the spleen. It was concluded that development of the thymus was most marked from 3 to 4 weeks of age and that both the thymus and spleen had matured by 9 weeks of age. (DOI: 10.1293/tox.25.55; J Toxicol Pathol 2012; 25: 55-61)
\end{abstract}

Key words: pleen, thymus, immunohistochemistry, weaning, development, SD rat

According to Immunotoxicity Studies For Human Pharmaceuticals (ICH S8 Guideline Step 5)1, evaluation of the potential adverse effects of human pharmaceuticals on the immune system should be incorporated into standard toxicity studies (STS). Among additional immunotoxicity studies, immunohistochemistry (IHC) of leukocyte populations can identify specific cell populations and may provide useful clinical biomarkers. IHC can be analyzed retrospectively if signs of immunotoxicity are observed in STS, and changes in cell types within a specific compartment of lymphoid tissue can be observed ${ }^{2}$. Marked changes in the numbers and proportions of tissue leukocytes (particularly $\mathrm{T}$ cells and $\mathrm{B}$ cells) in dogs and cats in the year following birth have been reported ${ }^{3-5}$. Moreover, immune function (morphological, cellular and biochemical changes) is known to decline with age in humans 6 , mice ${ }^{7}$, Fischer F344 rats $^{8}$, beagles ${ }^{9}$ and cynomolgus monkeys ${ }^{10}$. For these reasons, studies on age differences in the immune system are needed for the evaluation of immunotoxicity in neonatal or long-term toxicity studies. However, until now, detailed investigations of age differences in the immune organs have

Received: 14 June 2010, Accepted: 13 December 2011 Mailing address: Rinya Kodama, Drug Safety Research Laboratories, Shin Nippon Biomedical Laboratories, Ltd., 2438 Miyanoura, Kagoshima 891-1394, Japan

TEL: 81-99-294-2600 FAX: 81-99-294-3619

E-mail: kodama-rinya@snbl.co.jp

(C)2012 The Japanese Society of Toxicologic Pathology

This is an open-access article distributed under the terms of the Creative Commons Attribution Non-Commercial No Derivatives (by-ncnd) License $<$ http://creativecommons.org/licenses/by-nc-nd/3.0/>. not been performed in any species. Therefore, in the present study, we investigated changes in the chronological IHC phenotyping of immune cells in the thymus and spleen in $\mathrm{Crl}: \mathrm{CD}$ (SD) rats. This is one of the first studies to report on the chronological IHC phenotyping of immune cells in the thymus and spleen in SD rats.

Eighty-five male and eighty-three female SD rats (Charles River Laboratories Japan, Inc., Shiga, Japan) were used in this study. Neonates (38 males and 35 females) born to seven dams were evenly allocated to groups aged 1 to 4 weeks. They were housed with their dams from birth to 3 weeks and individually after weaning (from 4 weeks). All procedures involving animals were approved by the Institutional Animal Care and Use Committee of Shin Nippon Biomedical Laboratories Ltd. and were performed in accordance with standards published by the National Research Council (Guide for the Care and Use of Laboratory Animals, NIH OACU) and the U.S. National Institutes of Health policy on humane care and use of laboratory animals.

All rats were anesthetized by an intraperitoneal injection of sodium pentobarbital (Tokyo Chemical Industry Co., Ltd., Tokyo, Japan) solution $(6.48 \mathrm{mg} / \mathrm{mL}, 5 \mathrm{~mL} / \mathrm{kg})$ after being weighed (Fig. 1) and were then euthanized by exsanguination. Animals were necropsied chronologically up to the age of about one year (aged 1, 2, 3, 4, 9, 19, 23, 46 and 57 weeks), weekly from birth to 4 weeks of age and at subsequent points based on the end of the dosing or recovery period in repeated-dose toxicity studies. The thymuses and spleens from all animals were fixed in 10\% neutral buffered formalin. According to ICH S8 Guideline Step 51, histopathological changes in the thymus and spleen should be 


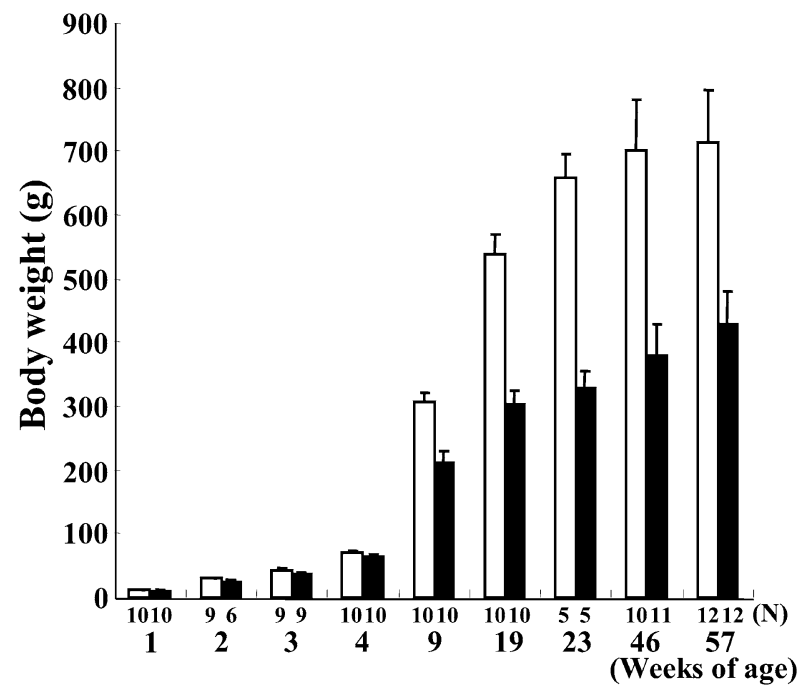

Fig. 1. Changes in body weight in male and female SD rats were graphed up to about one year of age. Neonates (38 males and 35 females) born to seven dams were evenly allocated to groups aged 1 to 4 weeks. They were housed with their dams from birth to 3 weeks of age and individually after weaning (from 4 weeks). White and black bars indicate males and females, respectively. $\mathrm{N}$ indicates the numbers of male and female SD rats. Values (g) are expressed as means $\pm \mathrm{SD}$

evaluated as indicators of systemic immunotoxicity. The thymus and spleen were selected because the former is a primary lymphoid organ considered to be consistently sensitive to morphological effects ${ }^{2}$, and the latter is a secondary lymphoid organ. They were incised vertically along the center, trimmed, embedded in paraffin and then sectioned. Three-micrometer sections were cut from formalin-fixed, paraffin-embedded blocks, mounted on positively charged glass slides and deparaffinized.

Consecutively cut sections were stained for IHC with CD3 (thymocytes, peripheral T lymphocytes and dendritic epidermal $\mathrm{T}$ cells), CD45RA (B cells among thoracic duct lymphocytes, with little labeling in bone marrow and none on thymocytes), CD68 (monocytes, macrophages and dendritic cells), CD161a (natural killer cell receptor protein 1a; NKR) and Proliferating Cell Nuclear Antigen (cycling cells during the last $5 \%$ of the G1 phase and the first $35 \%$ of the S phase; PCNA). All sections were then treated with N-Histofine Simple Stain Rat MAX PO (MULTI) (Nichirei Biosciences Inc., Tokyo, Japan). Immunoreactive elements were visualized by the DAB+ Liquid System (DAKO Japan, Kyoto, Japan). The morphometric analysis areas were the cortex and medulla of the thymus and the periarteriolar lymphoid sheath (PALS), marginal zone (MZ) and lymph follicle (LF) of the spleen. An IHC-positive reaction within a constant and tone range was assumed, and each IHC-positive cell (CD3-, CD45RA-, CD68-, NKR- and PCNA-positive cells expressed as T cells, B cells, macrophages, natural killer cells and proliferating cells, respectively, hereafter)
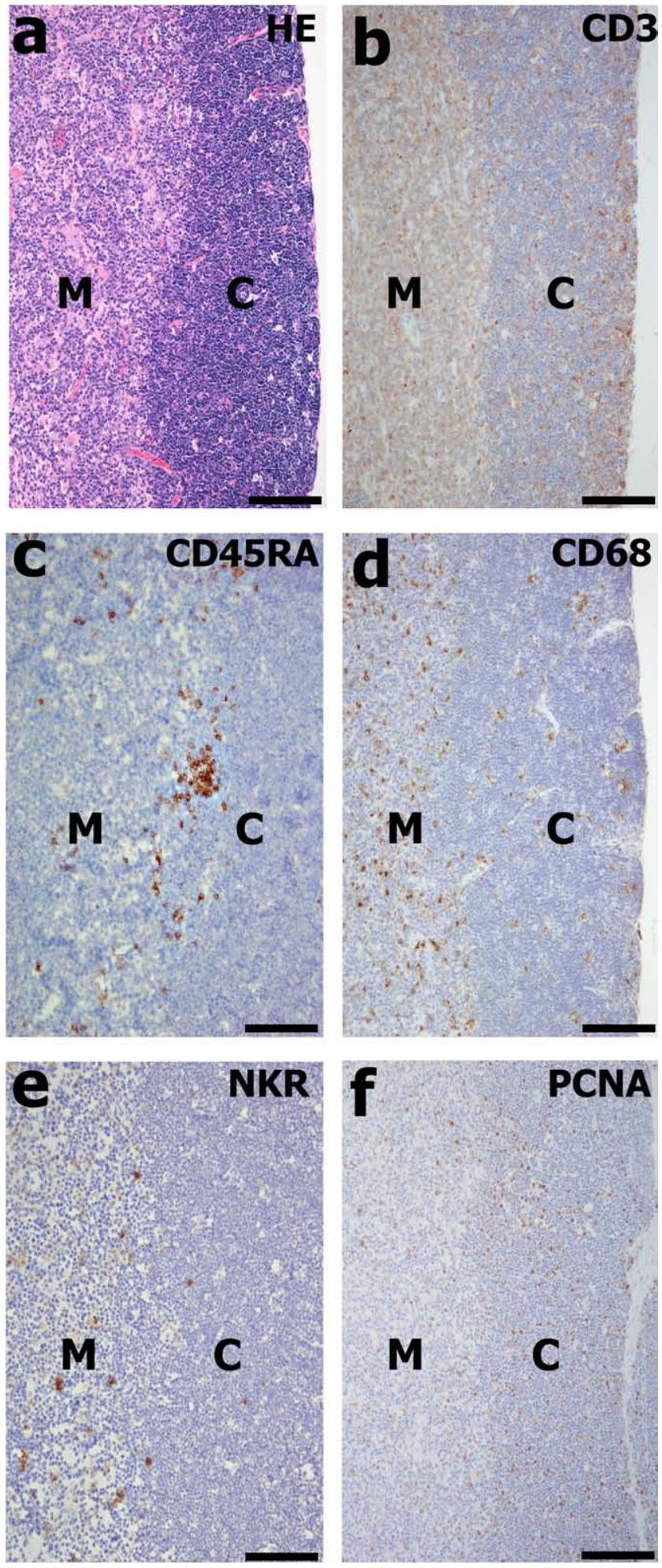

Fig. 2. Thymus histology and IHC from 9 weeks of age in male SD rats. a: HE stain. b: $\mathrm{T}$ cells diffusely locate in the cortex and medulla (CD3 IHC stain). c: A few B cells cluster at the corticomedullary junction (CD45RA IHC stain). d: A few macrophages were diffusely located in the cortex and medulla (CD68 IHC stain). e: A few natural killer cells were located around the corticomedullary junction (CD161a IHC stain). f: Proliferating cells are predominantly located in the cortex (PCNA IHC stain). Cortex (C) and medulla (M). Bar $=200$ $\mu \mathrm{m}$. 

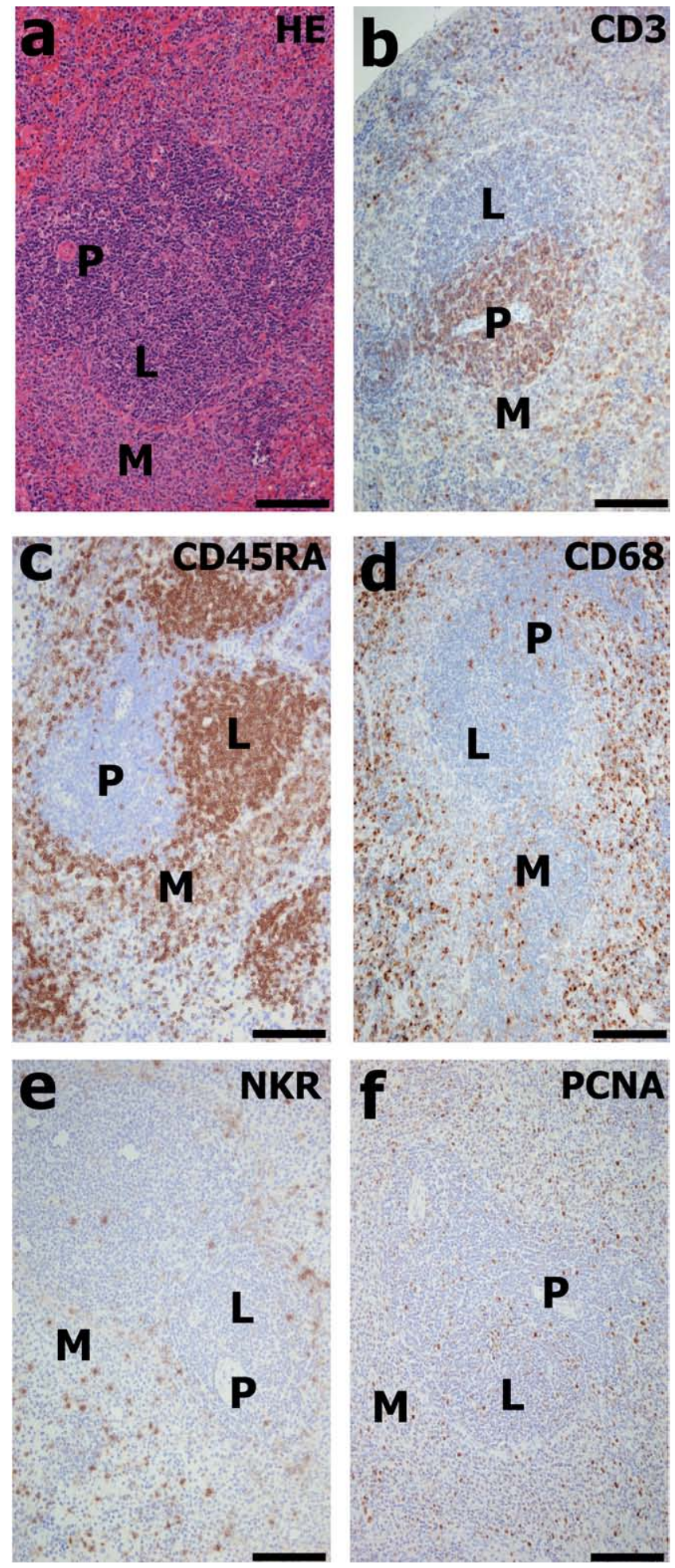

Fig. 3. Spleen histology and IHC from 9 weeks of age in male SD rats. a: HE stain. b: T cells are predominantly located in $\mathrm{P}$ and diffusely located in the red pulp (CD3 IHC stain). c: B cells are predominantly located in $\mathrm{L}$ and diffusely located in M (CD45RA IHC stain). A few macrophages are located approximately equally in all areas of the spleen (CD68 IHC stain). e: A few natural killer cells are located approximately equally in all areas of the spleen (CD161a IHC stain). f: Proliferating cells are predominantly located in P (PCNA IHC stain). Periarteriolar lymphoid sheath (P), lymph follicle (L) and marginal zone $(\mathrm{M})$. Bar $=200 \mu \mathrm{m}$. was picked up automatically and determined quantitatively using computerized image analyses (shown in Figs. 2 and 3) and morphometric techniques (Soft Imaging System GmbH, Muenster, Germany). The white pulp area and occupancy of the spleen were also calculated from these computerized image analyses and morphometric techniques. The data on $\mathrm{T}$ cells, B cells, macrophages, natural killer cells and proliferating cells obtained were compared with data at 9 weeks of age. We postulated that the immune system would have fully matured by that age because SD rats of that age, corresponding to an adolescent human and an age at which the nervous and reproductive systems have matured ${ }^{11,12}$, have been widely used in STS. Data were expressed as means \pm standard deviation (S.D.). Percentages against the preapplication values were analyzed for homogeneity of variance by the F-test. When the variances were homogenous, a Student's $t$-test was performed. When the variances were heterogeneous, the Aspin-Welch test was performed. These statistical analyses were conducted using MUSCOT statistical analysis software (Yukms Co., Ltd., Tokyo, Japan) at significance levels of $5 \%$ and $1 \%$.

Figure 4 shows changes in each cell in the cortex and medulla of the thymus in male and female SD rats. In the thymus, the number of T cells, B cells, macrophages, natural killer cells and proliferating cells was generally greater in the cortex than in the medulla through all age groups. $\mathrm{T}$ cells in the thymus increased until 4 weeks of age, and in particular, a marked increase of approximately three- to fourfold was noted from 3 to 4 weeks of age. T cells in the cortex markedly decreased from 4 to 9 weeks of age and gradually decreased thereafter. Atrophy of the cortex was observed in 1 of 5 males at 23 weeks of age and in almost all males and females at 46 and 57 weeks of age by HE staining. In the medulla, T cells decreased from 4 to 9 weeks of age, remained fairly stable until 23 weeks of age and decreased thereafter. The degree of the decrease after 4 weeks of age was less than that in the cortex. B cells in the thymus tended towards an increase with age. The degree of the increase at 57 weeks of age was greater in the cortex than in the medulla. Macrophages in the thymus increased from 3 to 4 weeks of age, remained fairly stable until 19 weeks of age and decreased thereafter. There was no marked difference in patterns between the cortex and medulla. Natural killer cells in the cortex showed high values triphasically at 1, 19 and 57 weeks of age. In the medulla, they increased from 3 or 4 weeks of age to 23 weeks of age and gradually decreased thereafter. Proliferating cells in the thymus increased until 4 weeks of age, decreased thereafter and showed a similar pattern to the number of $\mathrm{T}$ cells in the cortex.

In the spleen, the white pulp occupancy and area increased until 9 weeks of age and remained fairly stable thereafter (Fig. 5). Figure 6 shows changes in each cell in the PALS, MZ and LF of the spleen in male and female SD rats. At 4 weeks of age, the number of B cells, macrophages and proliferating cells in the MZ were much lower than the numbers at any other week. These results were considered to be due to the following: the LF was not apparent until 3 weeks 

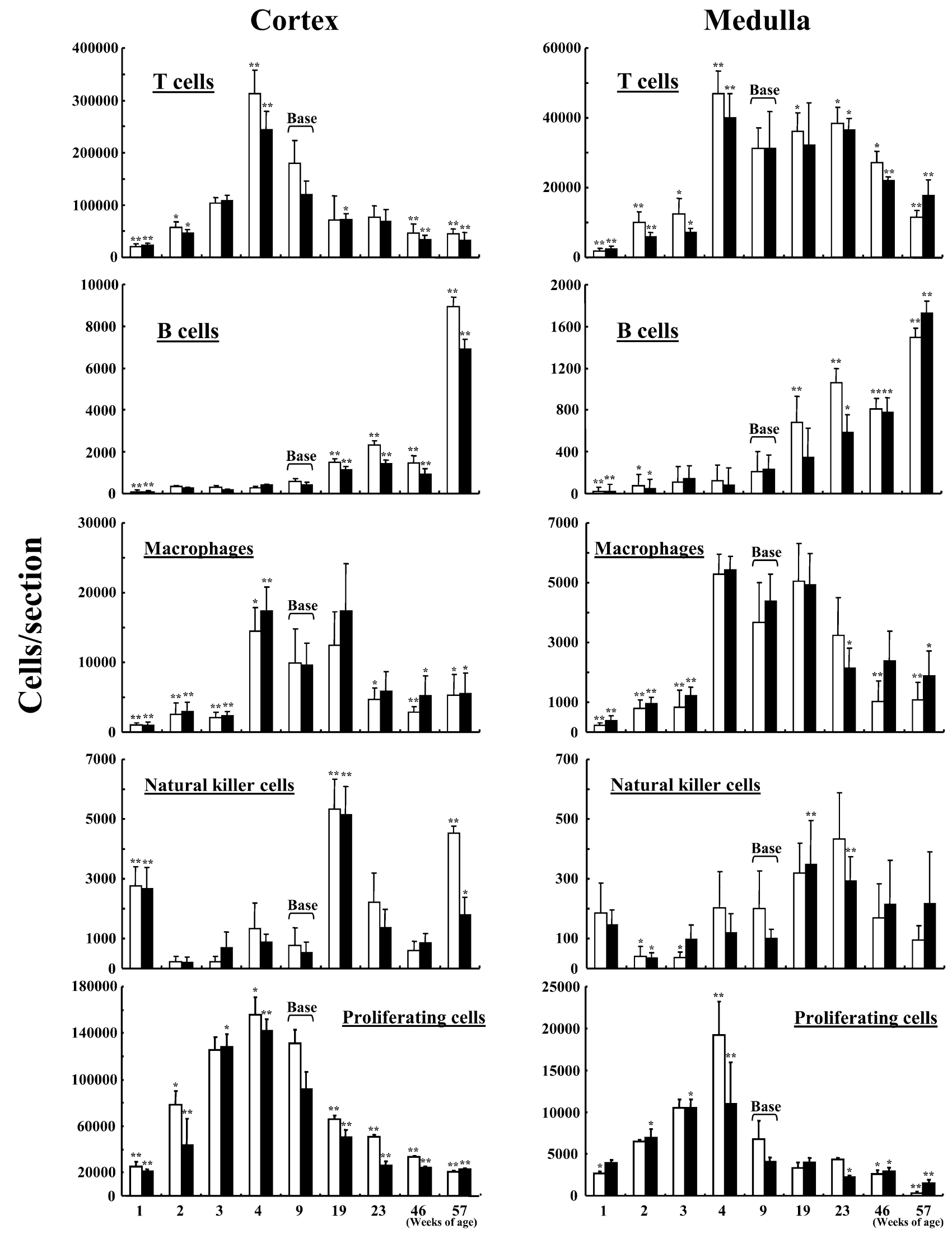

Fig. 4. Changes in T cells, B cells, macrophages, natural killer cells and proliferating cells in the cortex and medulla of the thymus of male and female SD rats. The number of each cell was graphed up to about one year of age. Cortex (left) and medulla (right). White and black bars indicate males and females, respectively. Values (cells) are expressed as means \pm SD. * and **: Asterisks indicate significant differences from the mean at 9 weeks of age ( $<<0.05$ and $p<0.01$, respectively). 

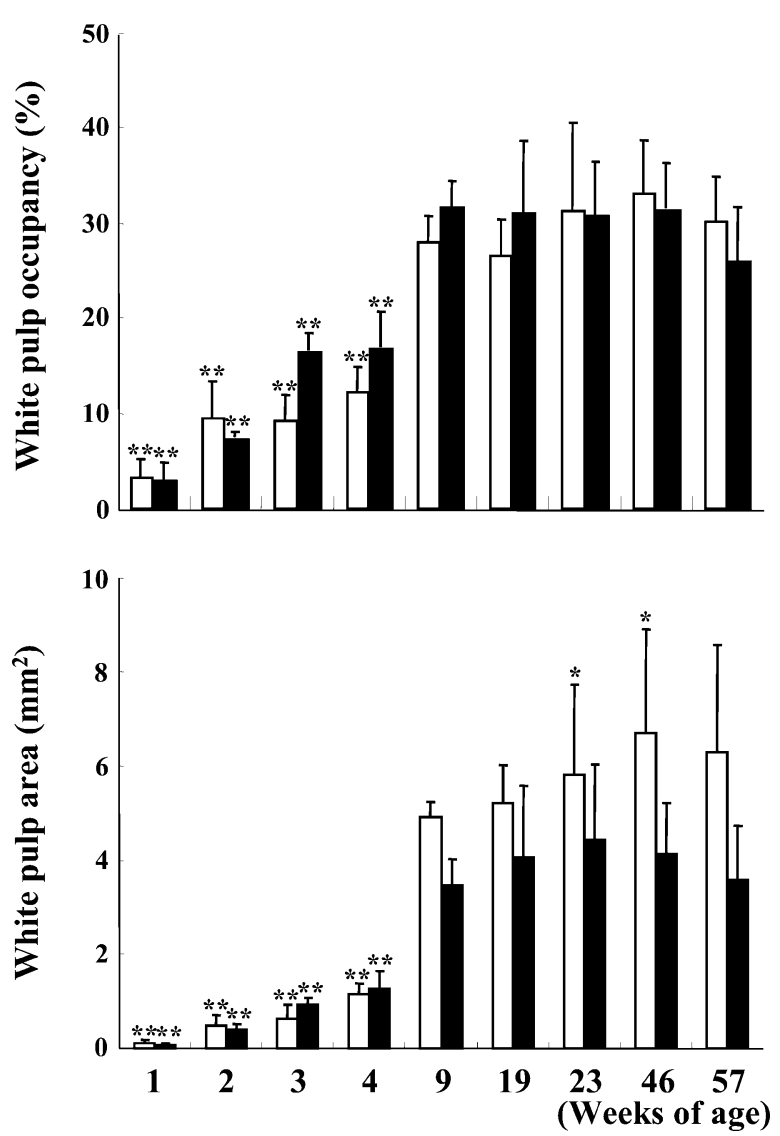

Fig. 5. White pulp occupancy (\%) and white pulp area $\left(\mathrm{mm}^{2}\right)$ in the spleen were graphed up to about one year of age in male and female SD rats. White and black bars indicate males and females, respectively. Values are expressed as means \pm SD. * and **: Asterisks indicate significant differences from the mean at 9 weeks of age $(p<0.05$ and $p$ $<0.01$, respectively).

of age but was clearly evident in the $M Z$ from 4 weeks of age; therefore, the LF was separated from the MZ and adopted as the morphometric area from 4 weeks of age. Large numbers of T cells were noted in the spleen in this order: the PALS, MZ and then LF. In the PALS and MZ, they gradually increased until 9 weeks of age and became almost flat thereafter. In the LF, they increased with age. B cells tended towards an increase with age in all areas of the spleen, and the largest number was found in the LF. The number of macrophages was approximately equal in all areas of the spleen and remained stable through all age groups. The smallest number of natural killer cells was noted in the PALS of the spleen, increasing at 9 weeks of age and becoming almost flat thereafter. In the MZ and LF, they increased biphasically at 19 and 57 weeks of age. Proliferating cells tended towards an increase until 19 weeks of age and gradually decreased thereafter in all areas of the spleen. The largest number was found in the LF.

The present study is one of the first to have investigated chronological changes in the IHC phenotyping of immune cells in the thymus and spleen in male and female SD rats up to the age of about one year. Moreover, we revealed differences in the characteristics of thymic and splenic development. In the thymus, $\mathrm{T}$ cells comprised the majority of the cellular components in the cortex and medulla, and their number increased markedly from 3 to 4 weeks of age (approximately 3 to 4 -fold). Proliferating cells also increased markedly at these points. In the present study, juveniles routinely suckled until 3 weeks of age and were weaned thereafter. Neonates have a T-helper (Th) 2-dominant immune system derived from maternal immunity. In juveniles, exogenous antigens are generally known to be kept at a low level by the mother-infant immune system in two ways: antigens in the blood are neutralized by transplacentally acquired $\operatorname{IgG}$, and those in the digestive tract are neutralized by $\operatorname{Ig} \mathrm{A}$ acquired from milk. After weaning, they actively ingest food, and exposure to exogenous antigens naturally increases ${ }^{4,5}$. It has been reported ${ }^{13}$ that intestinal microbiota is postulated to be a posterior immunomodulator that is an inverse Th2-dominant immune system in juveniles derived from maternal immunity. It was considered, therefore, that active food ingestion increased the exposure of juveniles to exogenous antigens and activated the intestinal microbiota. Activated microbiota modulated the Th1-dominant immune system (ingestant desensitization ${ }^{13}$ ) and resulted in differentiation and maturation of immature T cells. Weaning is considered to trigger marked $\mathrm{T}$ cell proliferation.

In the spleen, the white pulp area and occupancy increased until 9 weeks of age and remained fairly stable thereafter. Moreover, the IHC phenotyping showed that the number of cells composing each area in the spleen (PALS, $\mathrm{MZ}$ or LF) increased markedly from 4 to 9 weeks of age. However, there was a slight increase in accompanying cell proliferation at this point. These results were considered to indicate that mature $\mathrm{T}$ cells released from the thymus contributed to the increase in the number of T cells in the PALS of the spleen. T cells in the thymus tended to regress after 9 weeks of age; in contrast, their number in each area of the spleen was maintained. It was considered that an age-related decline was unlikely to have occurred in the secondary lymphoid organs because of an influx from the other lymphoid organs at 4 weeks of age and proliferation in the PALS ${ }^{14}$. $\mathrm{B}$ cells tended to increase with age in the spleen as well as the thymus, which is consistent with age-related changes in humans ${ }^{14,15}$. It has been reported that $\mathrm{T}$ cell function and recruiting of naïve $\mathrm{T}$ cells decline with age ${ }^{15}$. Age-related enhancement of humoral immunity and the nonspecific immune response may be compensatory reactions to declined specific cellular immunity.

From the above, it was concluded that development of the thymus, the primary lymphoid organ, was most marked from 3 to 4 weeks of age, earlier than the spleen, the secondary lymphoid organ, and that both the thymus and spleen had matured by 9 weeks of age. It was suggested that weaning, an external factor, was a possible trigger for the initiation of development of the thymus from 3 to 4 weeks of age and that mature $\mathrm{T}$ cells released from the thymus and B cell proliferation in the LF contributed to maturation of the spleen 
PALS

MZ

LF
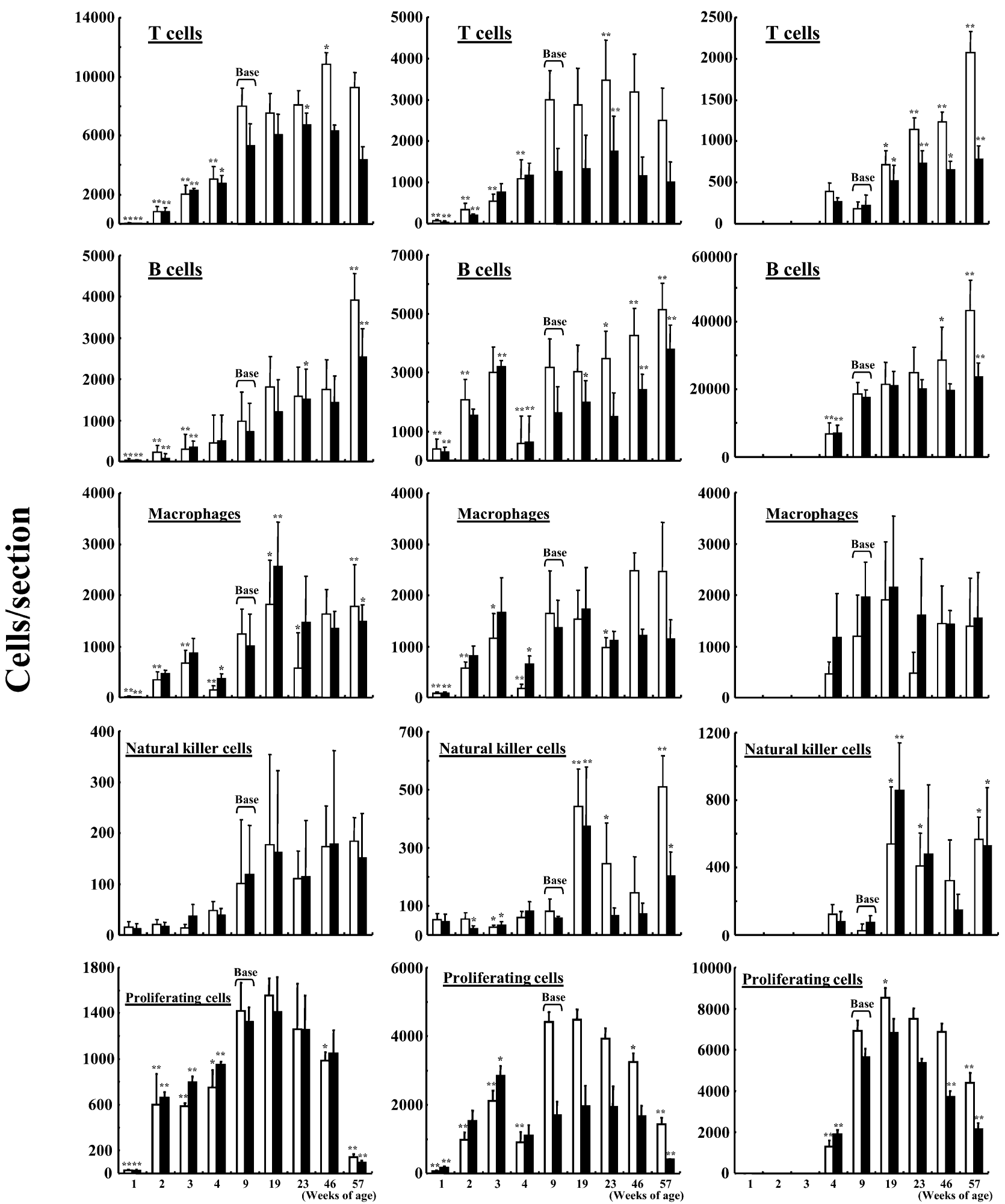

Fig. 6. Changes in T cells, B cells, macrophages, natural killer cells and proliferating cells in the periarteriolar lymphoid sheath (PALS), marginal zone (MZ) and lymph follicle (LF) of the spleen of male and female SD rats. The number of each cell was graphed up to about one year of age. At 4 weeks of age, the numbers of B cells, macrophages and proliferating cells in the MZ were much lower than those at any other week. These results were considered to be due to the following: the LF was not apparent until 3 weeks of age but was clearly evident in the MZ from 4 weeks of age; therefore, the LF was separated from the MZ and adopted as the morphometric area from 4 weeks of age. PALS (left), MZ (middle), and LF (right). White and black bars indicate males and females, respectively. Values (cells) are expressed as means \pm SD. * and **: Asterisks indicate significant differences from the mean at 9 weeks of age $(\mathrm{p}<0.05$ and $\mathrm{p}<0.01$, respectively). 
by 9 weeks of age. Moreover, it was concluded from these findings that when evaluating immunotoxicity in toxicity studies, in addition to the age and treatment duration, the weaning period must be also considered when distinguishing between the transient effects of immune modulation and the persistent consequences of developmental toxicity.

Acknowledgment: The authors are grateful to Medical English Service, K. K. for its review of the manuscript and advice.

\section{References}

1. Immunotoxicity Studies For Human Pharmaceuticals. ICH S8 Guideline Step 5.

2. Harleman JH. Approaches to the identification and recording of findings in the lymphoreticular organs indicative for immunotoxicity in regulatory type toxicity studies. Toxicology. 142: 213-219. 2000. [Medline] [CrossRef]

3. Day MJ. Immune system development in the dog and cat. J Comp Pathol. 137: S10-S15. 2007. [Medline] [CrossRef]

4. Morein B, Abusugra I, and Blomqvist G. Immunity in neonates. Vet Immunol Immunopathol. 87: 207-213. 2002. [Medline] [CrossRef]

5. Morein B, Blomqvist G, and Hu K. Immune responsiveness in the neonatal period. J Comp Pathol. 137: S27-S31. 2007. [Medline] [CrossRef]

6. Singh J, and Singh AK. Age-related changes in human thymus. Clin Exp Immunol. 37: 507-511. 1979. [Medline]
7. Schwander R, Hess MW, Keller HU, and Cottier H. The post-natal development of lymph nodes in mice. Immunobiology. 157: 425-436. 1980. [Medline] [CrossRef]

8. Cheung HT, and Nadakavukaren MJ. Age-dependent changes in the cellularity and ultrastructure of the spleen of Fischer F344 rats. Mech Ageing Dev. 22: 23-33. 1983. [Medline] [CrossRef]

9. Yang TJ, and Gawlak SL. Lymphoid organ weights and organ: body weight ratios of growing beagles. Lab Anim. 23: 143-146. 1989. [Medline] [CrossRef]

10. Spoor MS, Radi ZA, and Dunstan RW. Characterization of age- and gender-related changes in the spleen and thymus from control cynomolgus macaques used in toxicity studies. Toxicol Pathol. 36: 695-704. 2008. [Medline] [CrossRef]

11. Baldrick P. Developing drugs for pediatric use: a role for juvenile animal studies? Regul Toxicol Pharmacol. 39: 381389. 2004. [Medline] [CrossRef]

12. FDA 2006. Guidance for industry nonclinical safety evaluation of pediatric drug products. Document available at http://www.fda.gov/cder/guidance/index.htm.

13. Ji GE. Probiotics in primary prevention of atopic dermatitis. Forum Nutr. 61: 117-128. 2009. [Medline] [CrossRef]

14. Gruver AL, Hudson LL, and Sempowski GD. Immunosenescence of ageing. J Pathol. 211: 144-156. 2007. [Medline] [CrossRef]

15. Bhan AK, Reinherz EL, Poppema S, McCluskey RT, and Schlossman SF. Location of T cell and major histocompatible antigens in the human thymus. J Exp Med. 152: 771782. 1980. [Medline] [CrossRef] 